\title{
A Theoretical Approach to the Deposition of Cancer-Inducing Asbestos Fibers in the Human Respiratory Tract
}

\author{
Robert Sturm* \\ Department of Materials Science and Physics, Division of Physics, University of Salzburg, Hellbrunnerstrasse 34, \\ A-5020 Salzburg, Austria
}

\begin{abstract}
In the study presented here a stochastic model predicting the deposition of variably shaped asbestos fibers in the human respiratory tract is introduced. Deposition calculations are commonly based on the concept of the aerodynamic diameter. Besides Brownian diffusion, inertial impaction, and sedimentation, also interception representing the capture of particles at the carinal ridges of single airway bifurcations is considered as main deposition mechanism for the computation of regional and local deposition fractions. Concerning total deposition in the human respiratory tract, fibers with a cylindrical diameter, termed $\mathrm{d}_{\mathrm{p}}$, smaller than $0.1 \mu \mathrm{m}$ exhibit lower deposition fractions than comparable spheres, whilst fibers with $d_{p}$ greater than $0.1 \mu \mathrm{m}$ show higher deposition fractions than spheres. The fiber aspect ratio $\beta$ has only an insignificant influence on total deposition, i.e. total deposition fractions of fibers with $\beta=10$ and $\beta=100$ differ by 2 to $10 \%$. Regarding regional deposition, the fiber diameter represents a controlling factor insofar, as fibrous particles with $d_{p}=0.1$ $\mu \mathrm{m}$ are preferably deposited in the bronchioles and alveoli, whereas fibers with $d_{p}=10 \mu \mathrm{m}$ are exclusively accumulated in the extrathoracic region. Only deposition behavior of fibers with $d_{p}=1 \mu \mathrm{m}$ is more complex, since valuable particle fractions deposit in all compartments of the lungs. Local (i.e. generation-by-generation) deposition of fibrous particles is characterized by a deposition peak at airway generation 19 in the case of fibers with $d_{p}=0.1 \mu \mathrm{m}$. The deposition maximum is subject to a continuous dislocation towards more proximal airway generations with increasing $d_{p}$. Therefore, particles with $\mathrm{d}_{\mathrm{p}}=10 \mu \mathrm{m}$ are chiefly deposited in the first three bronchial airway generations. Differences of fiber deposition between sitting and light-work breathing conditions may be evaluated is insignificant in most cases. Only fibrous particles with $d_{p}$ $=1 \mu \mathrm{m}$ significantly change their deposition behavior with increasing inhalative flow rate in the way that proximal deposition is remarkably enhanced at the cost of bronchiolar and alveolar deposition. In general, any increase of the inhalative flow rate $\mathrm{Q}$ causes a successive dislocation of fiber deposition from distal to proximal compartments of the human respiratory tract. The results obtained from the theoretical approach lead to the conclusion that thin fibers with variable length tend to deposit in the pulmonary region of the lung, where they represent a remarkable hazard for mesothelioma. Thick fibers are preferentially accumulated in the proximal bronchi and therefore may induce bronchial lung cancer (adenocarcinoma).
\end{abstract}

Keywords: Asbestos, lung deposition, Monte Carlo model, impaction, interception, particle trajectory, stochastic lung model, statistical weight method, aspect ratio, sitting breathing, light-work breathing, lung cancer.

\section{INTRODUCTION}

\section{General Aspects}

Asbestos fibers and fibers in general represent nonspherical, elongated particles that are characterized by a length greater than $5 \mu \mathrm{m}$ and a length-per-diameter ratio, termed aspect ratio $\beta$, greater than 3 [1-3]. Based on numerous medical studies carried out during the past decades (for an overview see [4]), asbestos fibers have to be classified as notorious occupational hazard. Thereby, any exposures to airborne particles of this specific type in the workplace evidently enhance the incidence of e.g. asbestosis, fibrosis, and lung cancer for the affected subjects [3]. Although in the building industry asbestos fibers have been largely replaced by alternative materials such as man-made vitreous fibers (MMVFs) in the mean-time, investigations and discussions

*Address correspondence to this author at the Brunnleitenweg 41, A-5061 Elsbethen/Salzburg, Austria; E-mail: Robert.Sturm@sbg.ac.at concerning the effect of fibrous materials on the human respiratory tract are still of remarkable importance [1].

Long-term exposure to asbestos fibers dramatically increases the risk of lung cancer, whereby in general two types of asbestos-induced carcinoma may be distinguished. The so-called mesothelioma is characterized by the development of malignant cells in the pleura, i.e. the outer lining of the lungs and internal chest wall $[5,6]$. Long thin fibers, no matter if asbestos or other fibrous minerals, are deposited to a high extent in the lung alveoli, from where they may reach the lung parenchyma via specific clearance routes $[4,7]$. The storage of these potent carcinogens in the parenchyma may result in the penetration of a certain fiber fraction to the visceral pleura and, as a consequence of that, to the pleural surface, where malignant mesothelial plaques consisting of a single layer of flattened cuboidal cells are formed [5]. The second type of lung cancer caused by the exposure to asbestos is the so-called adenocarcinoma which commonly develops in the bronchial or pulmonary lung epithelium and 
occurs in conjunction with asbestosis, a partly extreme scarring of the lung tissue [8]. As in the case of mesothelioma, the latency period between exposure to asbestos and development of asbestos-induced carcinoma may be on the order of two, three or even more decades. Both types of lung cancer described here occur in the same lung only in very rare cases. The reasons for this phenomenon are not completely decoded hitherto, but the chemical and physical quality of fibrous particles may play an essential role.

\section{Deposition of Fibers in the Human Respiratory Tract - State of the Art}

For a better understanding of the processes standing behind the development of asbestos-induced lung cancer, the transport and deposition behavior of inhaled fibrous materials in the human respiratory tract has to be decoded successively by experimental and, not less important, by theoretical studies. In the past fiber inhalation experiments were exclusively conducted on laboratory animals $[2,9]$ and obtained results were extrapolated to the human lungs. Further experiments were carried out by using specific replicas of the upper bronchial airways and studying fibrous particle behavior under different breathing conditions $[2,3]$. All these investigations came to the unique result that fiber deposition is mainly controlled by the fiber diameter, whilst fiber length has to be regarded as a less significant determinant in this context. Hence, extraordinarily long fibers with small diameters may penetrate to the lung alveoli, where they are accumulated and cause the phenomena described above [9]. In addition to the findings regarding the relationship between fiber geometry and intrapulmonary deposition experimental studies could demonstrate that, besides the three main deposition mechanisms for spherical particles (i.e. inertial impaction, sedimentation, Brownian diffusion) a fourth deposition force, termed interception, becomes highly significant for fibers (Fig. 1; $[4,10,11]$. Interception represents the deposition of fibers oriented approximately perpendicular to the inhaled air stream on the carinal ridges of single airway bifurcations and is mainly limited to the uppermost part of the tracheobronchial tree.

The hazard of an exposure to asbestos (or other fibers) chiefly depends upon two factors: 1) the sites of fibrous particle deposition in the human respiratory tract and 2) the fiber dimension distribution at these deposition sites [9, 1214]. By finding out the preferential deposition localities of variably sized and elongated fibers in the lungs, an invaluable progress in understanding the etiological process and determining the hazard of fiber-induced diseases could be achieved. However, theoretical models dealing with the deposition of anisometric particles provide a significant support for the accomplishment of this remarkable aim. Most of the theoretical approaches to the deposition of fibers in the lung airways published in the scientific literature hitherto are based on the concept of the aerodynamic diameter which is the diameter of a unit-density sphere exhibiting exactly the same settling behavior as the particle of interest [4]. Thereby, the effect of non-sphericity on particle kinetics is commonly expressed by the so-called dynamic shape factor, always taking values greater than 1 in the case of fibers $[15,16]$. In the past, theoretical determination of this factor was conducted by the application of different mathematical tech- niques such as the computation of particle mobilities parallel and perpendicular to the airflow $[16,17]$, the calculation of fractal dimensions [18] or the use of the so-called Geldard method [19]. All of these approaches provided very similar results regarding the order of magnitude of the dynamic shape factor.

\section{Aims of the Present Study}

The objectives of the contribution presented here are two-fold: First, the mathematical background necessary for the simulation of fibrous particle deposition in the human respiratory tract is introduced. Thereby, main interest is focused on the computation of appropriate dynamic shape factors and resulting aerodynamic diameters of fibers with different aspect ratios. The aerodynamic diameter concept is implemented into a stochastic particle transport and deposition model [20] which was subject to numerous validation processes in the past and therefore may be evaluated as highly reliable in its category. Second, various deposition scenarios of fibers are computed, and their values for an estimation of health hazards are discussed.

\section{MATERIALS AND METHODS}

\section{Mathematical Approach to Fiber Geometry}

For a theoretical approach to fiber deposition in the human respiratory tract the concept of the aerodynamic diameter was applied. This parameter, noted as $d_{a e}$, refers to the diameter of a unit-density sphere with identical aerodynamic characteristics as the particle of interest. Mathematically, $d_{a e}$ may be written as follows [18, 21, 22]:

$$
d_{a e}=d_{e v} \cdot \sqrt{\frac{1}{\chi} \cdot \frac{C_{C}\left(d_{e v}\right)}{C_{C}\left(d_{a e}\right)} \cdot \frac{\rho_{p}}{\rho_{0}}} .
$$

In equation (1) $d_{e v}, \chi, C_{c}\left(d_{e v}\right), C_{c}\left(d_{a e}\right), \rho_{p}$, and $\rho_{0}$ denote, respectively, the equivalent volume diameter, the dynamic shape factor, the Cunningham slip correction factor for particles with $d_{\mathrm{ev}}$, the correction factor for particles with $d_{\mathrm{ae}}$, the density of the particle $\left(2.8 \mathrm{~g} \mathrm{~cm}^{-3}\right.$ in the case of asbestos fibers), and unit density $\left(1.0 \mathrm{~g} \mathrm{~cm}^{-3}\right)$. The equivalent volume diameter, $d_{e v}$, represents the diameter of a sphere with the identical volume as the fibrous particle considered for deposition calculation. In the case of a fiber with perfect cylindrical shape (Fig. 2), $d_{\mathrm{ev}}$ may be derived from the following formula:

$$
d_{e v}=\sqrt[3]{1.5 \cdot d_{p}^{3} \cdot \beta} .
$$

Here, $d_{p}$ represents the cylindrical particle diameter and $\beta$ the aspect ratio, i. e. the ratio between length and diameter of the fiber.

The dynamic shape factor, $\chi$, may be regarded as a dimensionless constant that brings the drag force experienced by an irregularly shaped particle moving in air in relation to the particle's equivalent volume diameter [4]. For a cylindrical fiber that is transported with random orientation through the airway tubes of the tracheobronchial tree, $\chi$ can be written in the following form $[2,3]$ :

$$
\frac{1}{\chi}=\frac{1}{3 \chi_{\|}}+\frac{2}{3 \chi_{\perp}} \text {. }
$$




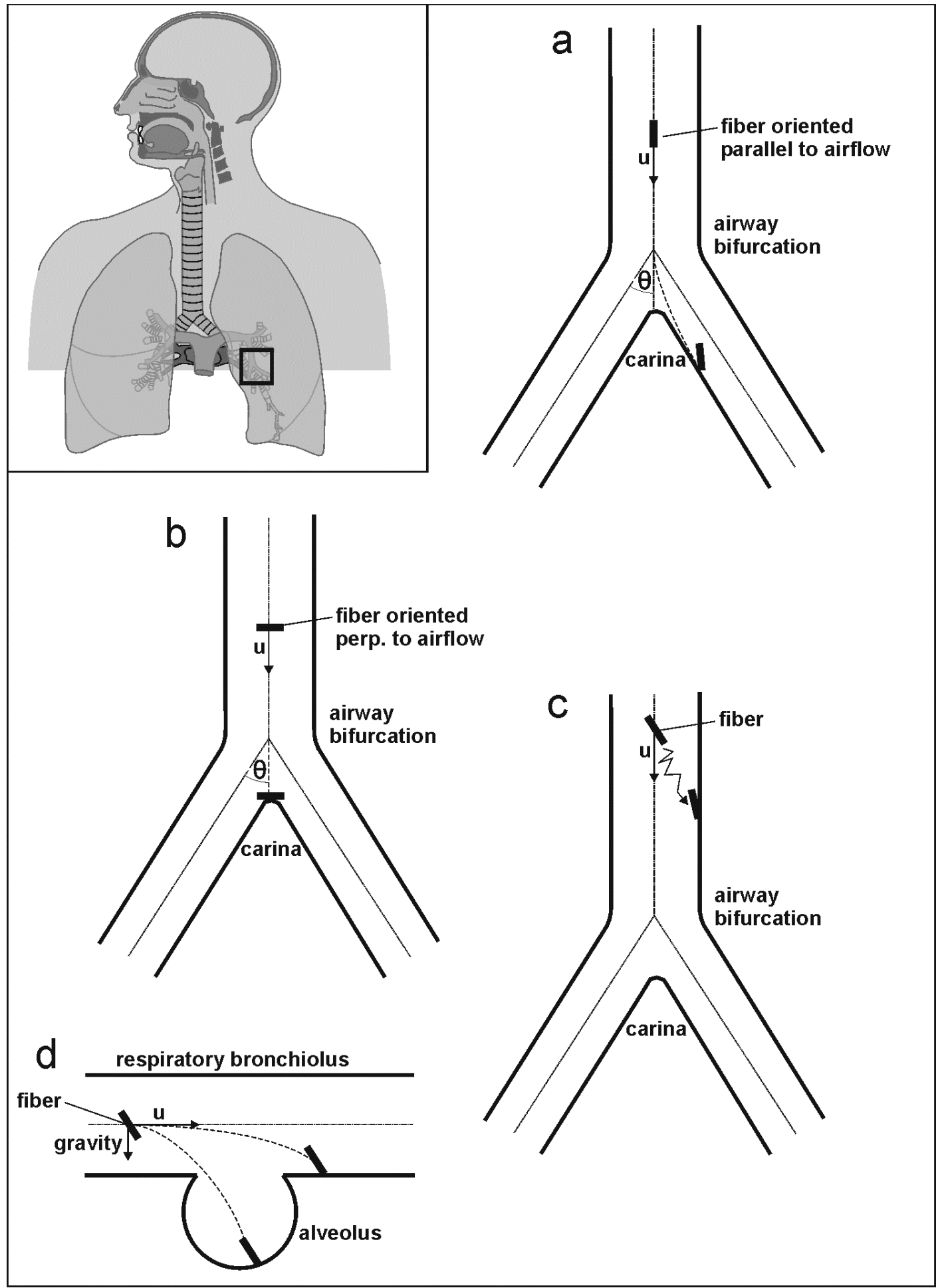

Fig. (1). Main mechanisms of fiber deposition in the human respiratory tract: (a) inertial impaction, (b) interception, (c) Brownian diffusion, (d) sedimentation. Further abbreviations: u...velocity of the particle in the respective airway, $\theta$...half branching angle of the airway bifurcation.

In the formula noted above, $\chi_{/}$is the dynamic shape factor of a given fiber oriented parallel to the direction of flow, whereas $\chi_{\_}$represents the dynamic shape factor of the fiber oriented perpendicular to the flow vector. The two factors are defined by the following equations [16]:

$$
\chi_{/ /}=\frac{\frac{4}{3} \cdot\left(\beta^{2}-1\right) \cdot \beta^{-\frac{1}{3}}}{\left\{\frac{\left(2 \beta^{2}-1\right)}{\sqrt{\beta^{2}-1}} \cdot \ln \left[\beta+\sqrt{\beta^{2}-1}\right]-\beta\right\}},
$$




$$
\chi_{\perp}=\frac{\frac{8}{3} \cdot\left(\beta^{2}-1\right) \cdot \beta^{-\frac{1}{3}}}{\left\{\frac{\left(2 \beta^{2}-3\right)}{\sqrt{\beta^{2}-1}} \cdot \ln \left[\beta+\sqrt{\beta^{2}-1}\right]+\beta\right\}} .
$$

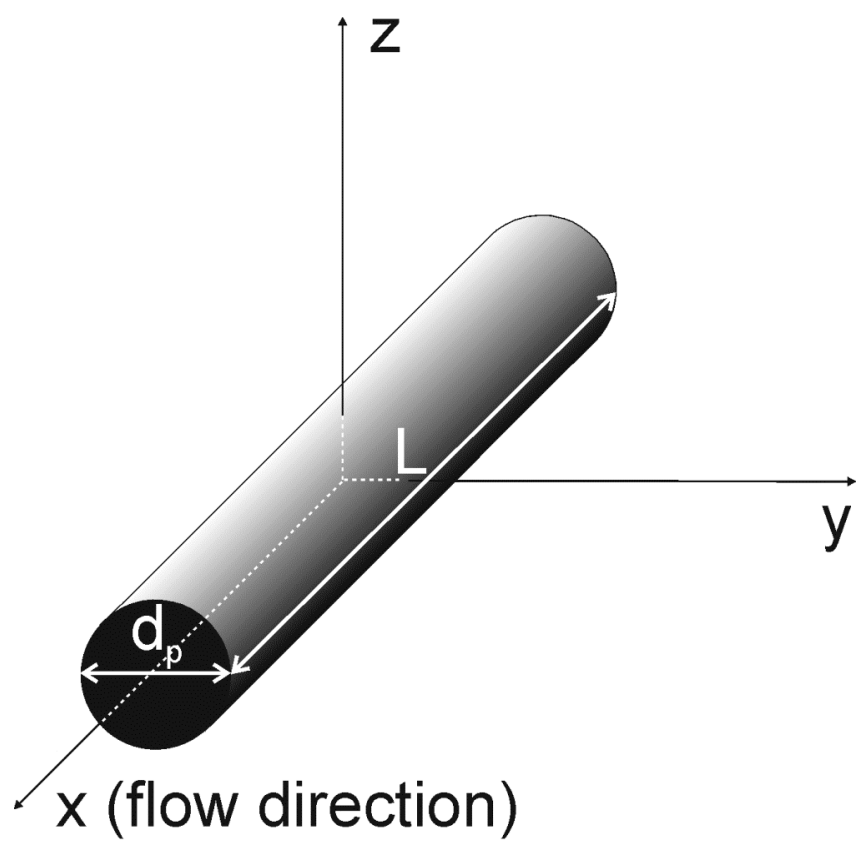

Fig. (2). Ideal cylindrical fiber used for the model calculations. Major geometric characteristics include the fiber diameter, termed $d_{p}$, the fiber length, termed $L$, as well as the aspect ratio, termed $\beta$, which is computed according to the formula: $\beta=L / d_{p}$.

The Cunningham slip correction factor, $\mathrm{C}_{\mathrm{c}}$, takes in account the circumstance that small particles $(<1 \mu \mathrm{m})$ are characterized by a faster settling than predicted by Stokes law. Contrary to the ideal assumptions of this law, the relative velocity of the gas right at the particle's surface significantly deviates from zero. Supposing respective air conditions of the human respiratory tract, i. e. $100 \%$ relative humidity, $76 \mathrm{~cm} \mathrm{Hg}$ atmospheric pressure, an air temperature of $37{ }^{\circ} \mathrm{C}$, and a related mean free path of the air molecules of $0.0712 \mu \mathrm{m}, \mathrm{C}_{\mathrm{c}}$ may be calculated according to the formula [4]:

$$
C_{C}=1+\frac{1}{76 d} \cdot[13.571+4.312 \cdot \exp (-7.7672 d)] \text {. }
$$

In Equation (6), $\mathrm{d}$ refers to a selected particle diameter $\left(\mathrm{d}_{\mathrm{ae}}\right.$ or $\left.\mathrm{d}_{\mathrm{ev}}\right)$.

\section{Simulation of Particle Transport and Deposition in the Human Respiratory Tract}

For particle deposition calculations the model provided by Koblinger \& Hofmann [20] was applied. This mathematical approach is based on the so-called random walk algorithm, according to which each inhaled particle is transported along a randomly selected path through the tracheobronchial and acinar regions. By application of the well established Monte Carlo simulation technique, large numbers of such particle trajectories (e. g. 10,000) may be generated to obtain statistically reliable data. Another improvement of the model with respect to approximations published more previously is the use of a stochastic lung architecture that provides a realistic variability of airway geometry and orientation within a specific lung generation. Morphometric data necessary for the generation of this probabilistic lung model were implemented into the approach, whereby respective data sets of the tracheobronchial tree [23] and the acinar region [24] were used. For each morphometric parameter the distribution of the measured values was determined and related probability density functions were created [25], with the help of which the stochastic lung was constructed airway by airway.

Computation of particle deposition was conducted by the application of specific deposition formulae for inertial impaction, sedimentation, and Brownian diffusion [4, 26]. Thereby, for each airway tube an individual deposition probability was computed. To account for interception representing an essential deposition force of fibers, correction factors for the bronchial airway generations 1 to 12 were computed which are based on numerical simulations of fiber behavior within single airway bifurcations [27-29] and are expressed as functions of Stokes number and aspect ratio.

To further improve the statistics of the Monte Carlo simulations, for each particle entering the lung multiple deposition scenarios were computed by application of the statistical weight method [20]. Here, a unit weight, which was attributed to the particle after intruding into the trachea, was multiplied by $\left(1-p_{i}\right)$ at each bifurcation with $p_{i}$ denoting the deposition probability at that bifurcation unit. The final deposition fraction in a specific airway bifurcation was given by the product of the actual weight and the respective deposition probability.

Deposition scenarios were created by assuming standard breathing conditions suggested by the ICRP [4] (sitting breathing: tidal volume of $750 \mathrm{ml}$, breathing frequency of 12 $\min ^{-1}$; light-work breathing: tidal volume of $1,250 \mathrm{ml}$, breathing frequency of $20 \mathrm{~min}^{-1}$ ).

\section{MODELING RESULTS}

\section{Aerodynamic Diameter and Total Deposition in the $\mathrm{Hu}$ - man Respiratory Tract}

Aerodynamic diameter $d_{a e}$ was calculated for fibers with $d_{p}$ ranging from 0.01 to $10 \mu \mathrm{m}$ and $\beta$ amounting to $3,10,50$, and 100 (Table 1). These dimensions sufficiently describe the geometries of inhalable fibers that may occur at the working place. In general, size distribution of airborne fibers may be described by a log-normal distribution with mean fiber length being on the order of several $\mu \mathrm{m}$ and fiber diameter ranging from 0.2 to $0.5 \mu \mathrm{m}$. As may be recognized from Table 1, fiber length partly has an insignificant effect on $\mathrm{d}_{\mathrm{ae}}$. Above all a doubling of $\beta$ from 50 to 100 only causes a slight enhancement of $d_{a e}$ which is commonly on the order of 5 to $10 \%$.

The effect of particle geometry on total deposition in the human respiratory tract, including both deposition in the extrathoracic region (ET) and particle accumulation in the thoracic region, is illustrated in Fig. (3), whereby asbestos fibers with a density of $2.8 \mathrm{~g} \mathrm{~cm}^{-3}$ as well as two values for $\beta$ $(10,100)$ were assumed. Concerning sitting breathing 
Table 1. Aerodynamic Diameters Calculated for Asbestos Fibers $\left(2.8 \mathrm{~g} \mathrm{~cm}^{-3}\right)$ with Different Cylindrical Diameters $d_{p}$ and Aspect Ratios $\beta$

\begin{tabular}{|c|c|c|c|c|}
\hline \multirow{2}{*}{$\mathbf{d}_{\mathbf{p}}(\boldsymbol{\mu \mathbf { m } )}$} & \multicolumn{4}{|c|}{ Aspect Ratio $\boldsymbol{\beta}$} \\
\cline { 2 - 5 } & $\mathbf{3}$ & $\mathbf{1 0}$ & $\mathbf{5 0}$ & $\mathbf{1 0 0}$ \\
\hline \hline 0.001 & 0.003 & 0.003 & 0.003 & 0.003 \\
\hline 0.002 & 0.005 & 0.006 & 0.006 & 0.006 \\
\hline 0.005 & 0.013 & 0.015 & 0.016 & 0.015 \\
\hline 0.010 & 0.026 & 0.030 & 0.031 & 0.031 \\
\hline 0.020 & 0.052 & 0.060 & 0.065 & 0.065 \\
\hline 0.050 & 0.129 & 0.153 & 0.173 & 0.179 \\
\hline 0.100 & 0.260 & 0.315 & 0.367 & 0.386 \\
\hline 0.200 & 0.524 & 0.644 & 0.768 & 0.814 \\
\hline 0.500 & 1.318 & 1.638 & 1.983 & 2.112 \\
\hline 1.000 & 2.641 & 3.299 & 4.016 & 4.287 \\
\hline 2.000 & 5.289 & 6.624 & 8.088 & 8.640 \\
\hline 3.000 & 7.937 & 9.949 & 12.161 & 12.995 \\
\hline 5.000 & 13.234 & 16.601 & 20.307 & 21.706 \\
\hline 10.000 & 26.475 & 33.231 & 40.674 & 43.484 \\
\hline
\end{tabular}

conditions (see above; [4]), fibers exhibit a lower total deposition than spheres for cylindrical particle diameters $d_{p}$ (Fig. 2) ranging from 0.001 to $0.1 \mu \mathrm{m}$ (Fig. 3a). For $d_{p}$ greater than $0.1 \mu \mathrm{m}$ total deposition of fibrous particles clearly exceeds the respective deposition of spheres. In general, total deposition within the noted range of $d_{p}$ follows a U-shaped curve with its minimum being located at $\mathrm{d}_{\mathrm{p}}=0.1 \mu \mathrm{m}$ as well as showing a maximum for ultrafine particles $(>80 \%)$ and for large particles $(100 \%)$. As already supposed by the computation of $\mathrm{d}_{\mathrm{ae}}$, fiber length has only a minor effect on total deposition for the assumed breathing conditions, whereby deposition discrepancies between the two fiber classes described here are on the order of 2 to $10 \%$. By changing the breathing conditions from sitting to light-work inhalation [4], only slight modifications of the total deposition curves may be recognized (Fig. 3b). Whilst deposition of ultrafine particles is largely independent of the increase in breathing frequency and tidal volume, large particles exhibit a more remarkable sensitiveness to the modified breathing conditions. This is chiefly reflected by an enhanced deposition for particles with $d_{p}$ greater than $1 \mu \mathrm{m}$. Furthermore, deposition differences between the two fiber classes partly reach some significance.

\section{Fiber Deposition in Different Lung Compartments}

Regarding regional fiber deposition, the human respiratory tract was subdivided into three compartments, i.e. the extrathoracic region, termed ET, the bronchial region, and the bronchiolar/alveolar region (Fig. 4). For this computation fibrous particles with $d_{p}$ amounting to $0.1 \mu \mathrm{m}, 1 \mu \mathrm{m}$, and 10 $\mu \mathrm{m}$ were considered, whereas for $\beta$ two different values (10, 100) were supposed again. Under the assumption of sitting
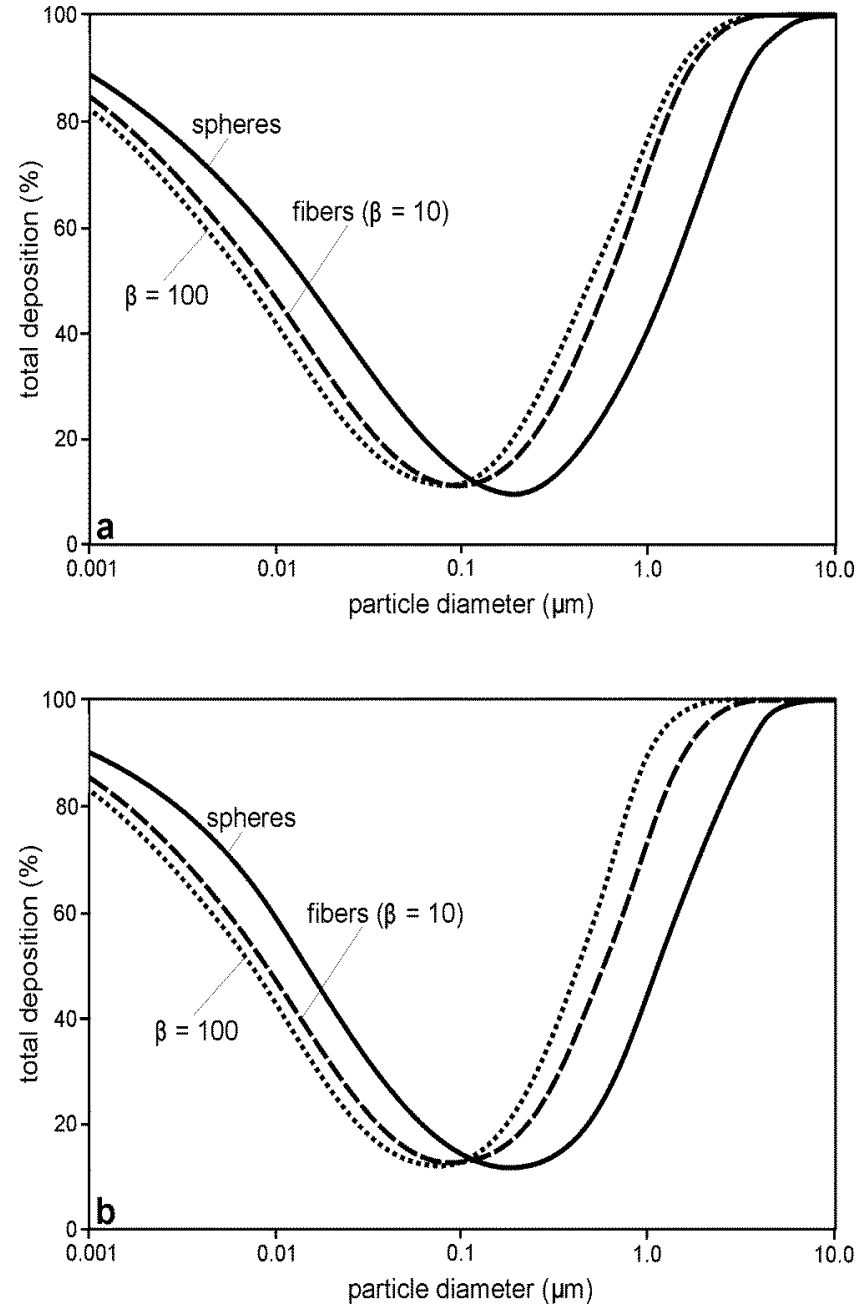

Fig. (3). Total deposition of spheres and asbestos fibers with $\beta=10$ and $\beta=100$ in the human respiratory tract: (a) deposition curves obtained for sitting breathing conditions, (b) deposition curves obtained for light-work breathing conditions [4].

breathing conditions (Fig. 4a-c), the three size classes show a totally different regional deposition behavior. Whilst fibers with $\mathrm{d}_{\mathrm{p}}=0.1 \mu \mathrm{m}$ are mainly deposited in bronchiolar and alveolar lung region ( 70 to $80 \%$; Fig. 4a), respective deposition of fibers with $d_{p}=1 \mu \mathrm{m}$ increasingly depends upon $\beta$. Besides the deposition maximum in the bronchiolar and alveolar lung region, fibrous particles with $\beta=10$ are preferably accumulated in the bronchial region (about $30 \%$ ), whereas fibers with $\beta=100$ are chiefly deposited in the extrathoracic region (about $35 \%$; Fig. 4b). Particles with $d_{p}$ $=10 \mu \mathrm{m}$ are completely filtered in the mouth cavity and oropharynx, thereby having no chance to penetrate to the pulmonary compartment (Fig. 4c). Concerning light-work breathing conditions (Fig. 4d-f), only regional deposition of fibers with $d_{p}=1 \mu \mathrm{m}$ is subject to significant modifications with respect to the deposition under sitting breathing. For fibrous particles with $\beta=10$ maximum deposition is again located in the bronchiolar and alveolar compartment, but extrathoracic and bronchial deposition is remarkably enhanced now. Fibers with $\beta=100$, on the other side, are preferentially accumulated in the extrathoracic region $(60 \%)$ and penetrate to the terminal lung compartment in low amounts (about $5 \%$; Fig. 4f). 

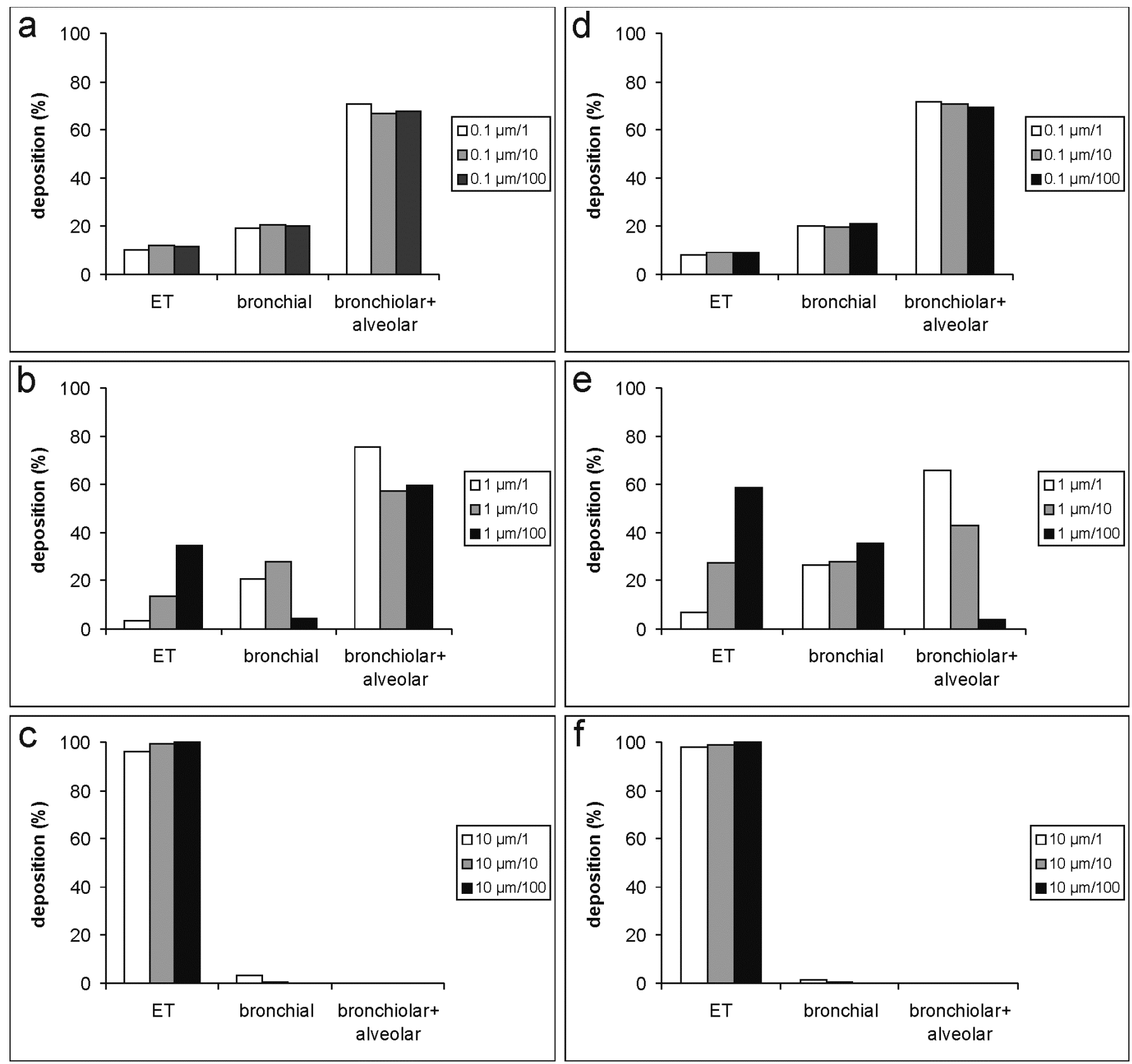

Fig. (4). Deposition of spheres $(\beta=1)$ and fibers $(\beta: 10$ and 100) with different cylindrical diameters in three lung regions, i.e. the extrathoracic region, the bronchi, and the bronchiolar/ alveolar region: (a) $d_{p}=0.1 \mu \mathrm{m}$, sitting breathing, (b) $d_{p}=1 \mu \mathrm{m}$, sitting breathing, (c) $d_{p}=10$ $\mu \mathrm{m}$, sitting breathing, (d) $d_{p}=0.1 \mu \mathrm{m}$ light-work breathing, (e) $d_{p}=1 \mu \mathrm{m}$, light-work breathing, (f) $d_{p}=10 \mu \mathrm{m}$, light-work breathing.

\section{Deposition of Fibers in Various Airway Generations of the Lungs}

As deposition of fibrous material in single airway generations of the lungs represents a valuable parameter for the estimation of fiber-related health hazards, respective deposition-by-generation diagrams were computed and are depicted in Fig. (5). Concerning both the geometric particle properties and the breathing conditions, identical assumptions as for the simulation of regional deposition were made. Under sitting breathing conditions (Fig. 5a-c), fibers with $d_{p}=0.1 \mu \mathrm{m}$ exhibit deposition maxima at airway generation 19 (Fig. 5a). For fibrous particles with $d_{p}=1 \mu \mathrm{m}$ deposition maxima may be recognized at the same airway generation, whereby differences of local deposition between the two fiber classes ( $\beta$
$=10, \beta=100)$ become more significant. Additionally, proximal deposition, i.e. deposition in the airway generations 0 (trachea) to 18 , becomes remarkably enhanced (Fig. 5b). Fibers with $d_{p}=10 \mu \mathrm{m}$ are mainly accumulated in the first two airway generations of the tracheobronchial tree, whereas deposition in more distal airways may be evaluated as nearly negligible (Fig. 5c). By changing inhalation conditions from sitting to light-work breathing (Fig. 5d-f), significant modifications of the deposition patterns may be only recognized for those fibrous particles with $d_{p}=1 \mu \mathrm{m}$ (Fig. 5e). Here, terminal deposition maxima become decreased at the cost of an increased particle deposition in the more proximal airway generations. With increasing $\beta$ deposition of inhaled fibers is successively dislocated to the trachea and the bronchi. 

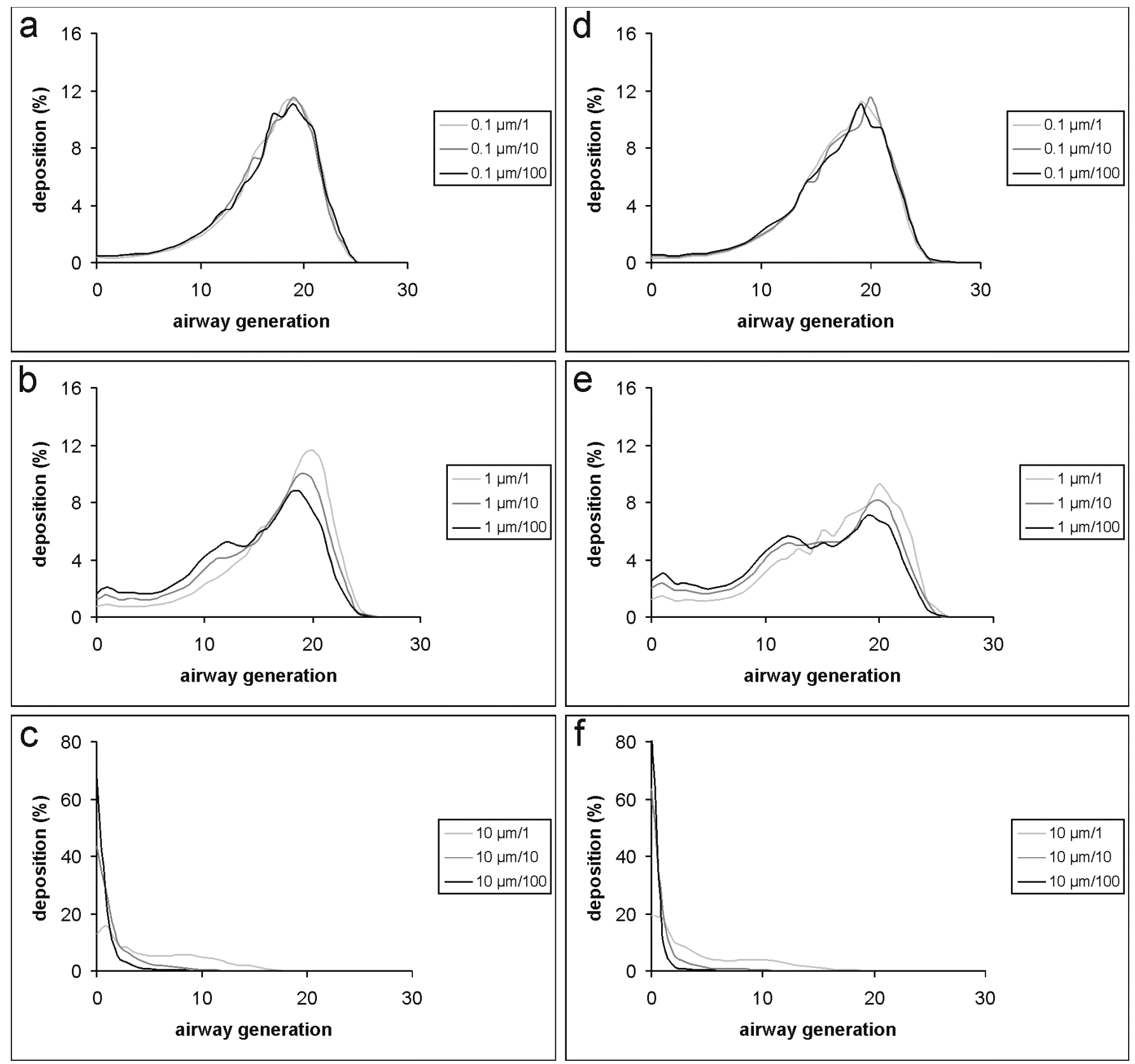

Fig. (5). Deposition of spheres $(\beta=1)$ and fibers ( $\beta$ : 10 and 100) with different cylindrical diameters in the single airway generations of the lungs: (a) $d_{p}=0.1 \mu \mathrm{m}$, sitting breathing, (b) $d_{p}=1 \mu \mathrm{m}$, sitting breathing, (c) $d_{p}=10 \mu \mathrm{m}$, sitting breathing, (d) $d_{p}=0.1 \mu \mathrm{m}$ light-work breathing, (e) $d_{p}=1 \mu \mathrm{m}$, light-work breathing, (f) $d_{p}=10 \mu \mathrm{m}$, light-work breathing.

The essential role of fibrous particles with $d_{p}=1 \mu \mathrm{m}$ and their deposition in the human lungs is additionally illustrated in Fig. (6), where the uppermost part of the human respiratory tract is graphically subdivided into seven regions, and deposition fractions in each of these regions are represented by respective grey shades. As exhibited by the single images, $\beta$ has a noticeable effect on fiber deposition in the single compartments insofar, as under sitting breathing conditions (Fig. 6a) an enhancement of $\beta$ from 10 to 100 causes a dislocation of deposited mass from more distal to more proximal lung generations. This important circumstance is further pronounced under light-work breathing conditions (Fig. 6b).

\section{Dependence of Fiber Deposition on Particle Geometry and Airflow Properties}

The dependence of particle deposition upon the inhalative flow is appropriately demonstrated by introducing the product $\mathrm{d}_{\mathrm{ae}}{ }^{2} * \mathrm{Q}$, where $\mathrm{Q}$ denotes the inhalative flow rate with the common dimension $\mathrm{cm}^{3} \mathrm{~min}^{-1}$ (Fig. 7). Any increase of $\mathrm{d}_{\mathrm{ae}}{ }^{2} * \mathrm{Q}$ is either caused by an enhancement of $\mathrm{d}_{\mathrm{ae}}$, an enhancement of $\mathrm{Q}$ or an increase of both physical parameters. In the concrete case, $\mathrm{Q}$ was successively enhanced from $7,500 \mathrm{~cm}^{3} \mathrm{~min}^{-1}$ (sleeping breathing) to $60,000 \mathrm{~cm}^{3} \mathrm{~min}^{-1}$ (hard-work breathing). For $\mathrm{d}_{\mathrm{ae}}{ }^{2} * \mathrm{Q}$ ranging from $10^{-3} \mu \mathrm{m}^{2}$ $\mathrm{cm}^{3} \mathrm{~min}^{-1}$ to $10^{5} \mu \mathrm{m}^{2} \mathrm{~cm}^{3} \mathrm{~min}^{-1}$, fiber deposition in the 


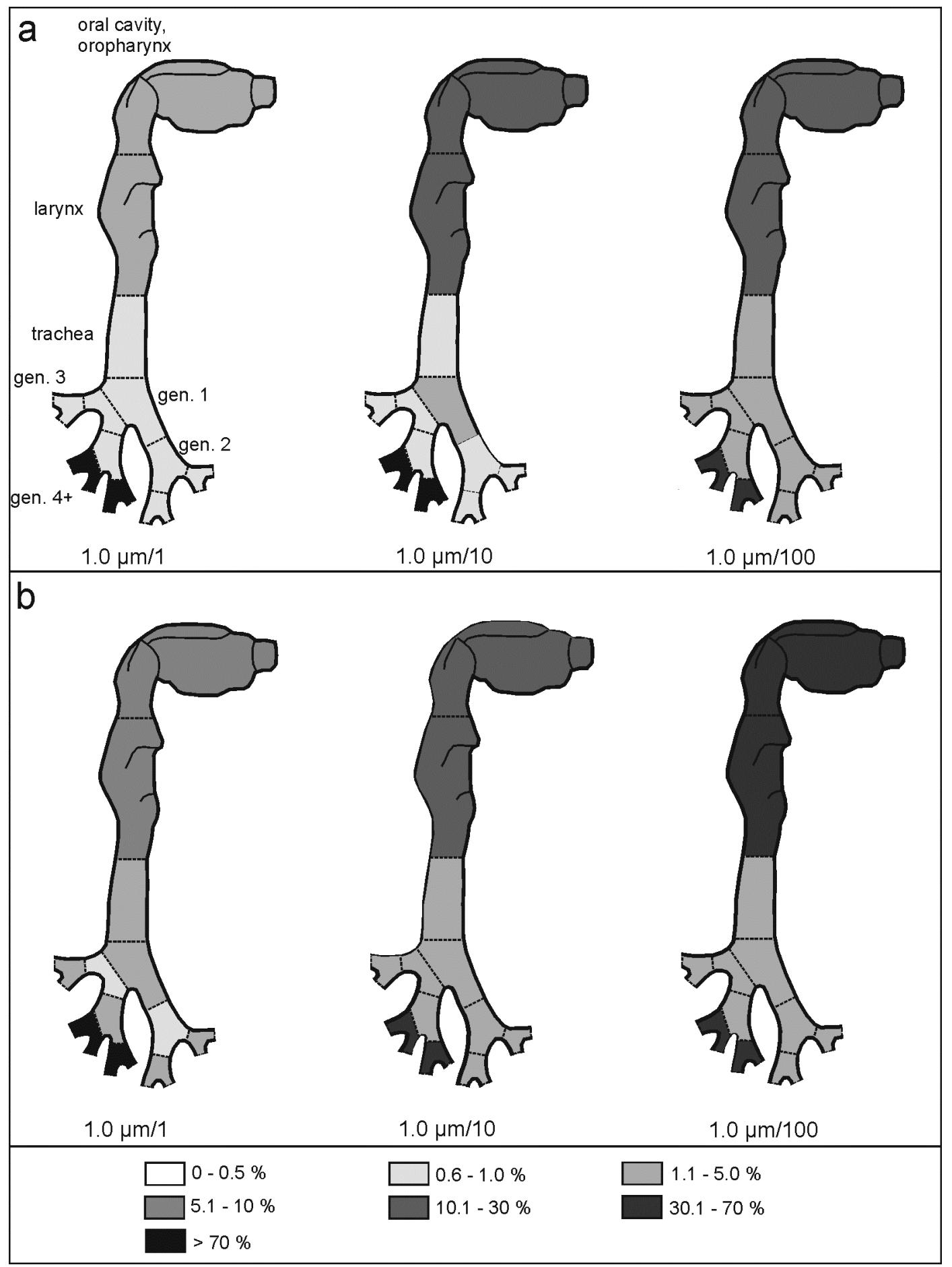

Fig. (6). Graphical presentation of particle deposition ( $\beta: 1,10$ and 100) in the extrathoracic region (oral cavity, oropharynx, larynx) and the uppermost airways of the tracheobronchial tree [2,3]: (a) sitting breathing conditions, (b) light-work breathing conditions [4].

extrathoracic region approximately follows a sigmoidal function, thereby reaching $100 \%$ for $\mathrm{d}_{\mathrm{ae}}{ }^{2} * \mathrm{Q}$ greater than $10^{3}$ $\mu \mathrm{m}^{2} \mathrm{~cm}^{3} \mathrm{~min}^{-1}$ (Fig. 7a). In the trachea and the following airway generations 1 to 3 maximum deposition, commonly being on the order of 1 to $1.5 \%$, occurs at $\mathrm{d}_{\mathrm{ae}}{ }^{2} * \mathrm{Q}=10^{3} \mu \mathrm{m}^{2}$ $\mathrm{cm}^{3} \min ^{-1}$, whilst for lower and higher values of $\mathrm{d}_{\mathrm{ae}}{ }^{2} * \mathrm{Q}$ deposition is subject to a dramatic decline. A further enhancement of fiber accumulation in the respective airways can be observed at $\mathrm{d}_{\mathrm{ae}}{ }^{2} * \mathrm{Q}=10^{-3} \mu^{2} \mathrm{~cm}^{3} \min ^{-1}$ (Fig. 7b-e). In airway generation $4+$, representing airway generation 4 itself and all the following lung generations, maximum deposition may be recognized at low and intermediate values of $\mathrm{d}_{\mathrm{ae}}{ }^{2} * \mathrm{Q}$ (Fig. 7f). Thereby, deposition at the left maximum exceeds $60 \%$, whereas the right peak reaches deposition values that range from 25 to $45 \%$.

\section{DISCUSSION AND CONCLUSIONS}

For an appropriate evaluation of health hazards emanating from the exposure to asbestos fibers, a comprehensive 

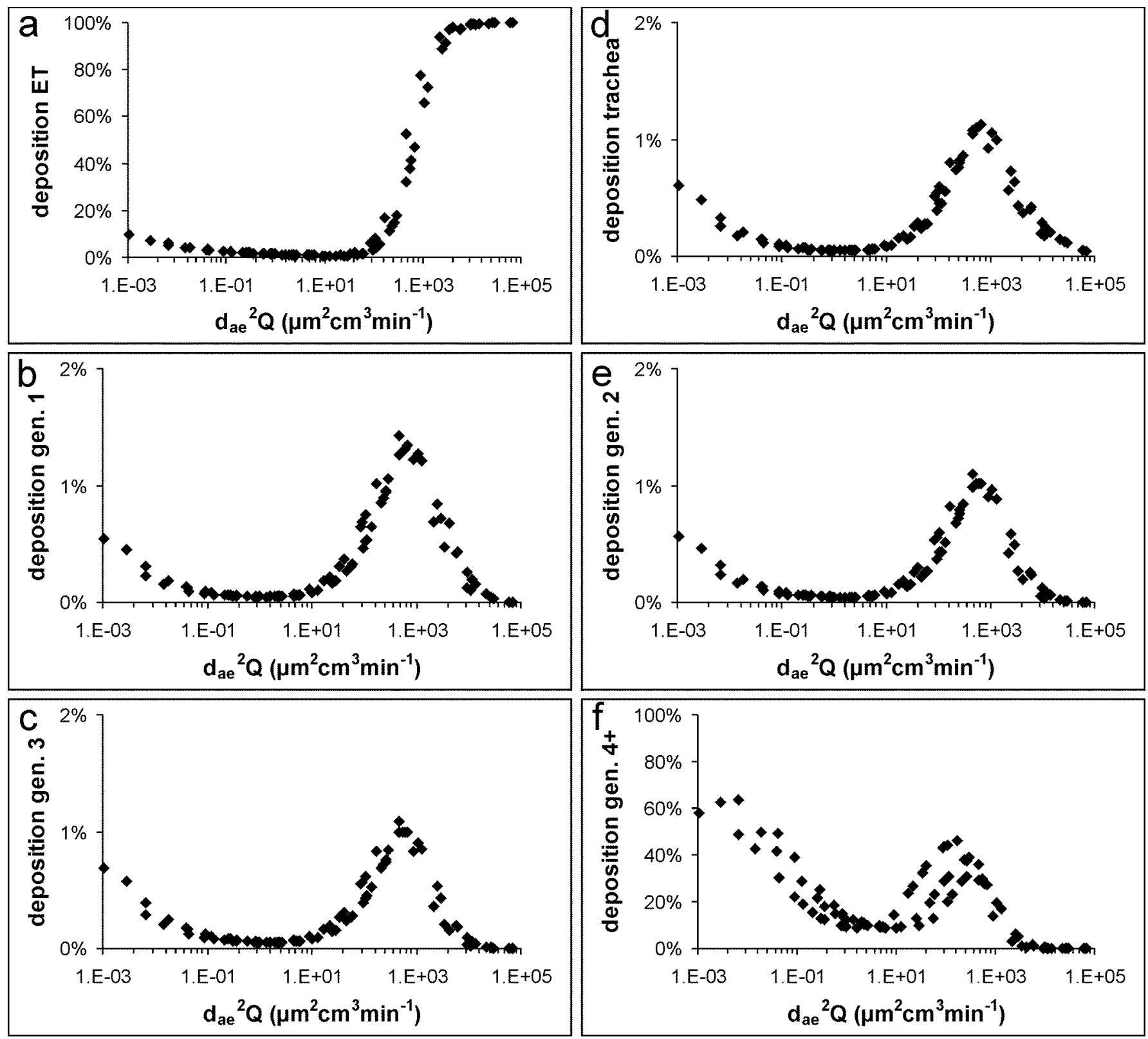

Fig. (7). Dependence of particle deposition in different parts of the lungs on the product $\mathrm{d}_{\mathrm{ae}}{ }^{2} * \mathrm{Q}$, with $\mathrm{d}_{\mathrm{ae}}$ including geometric particle properties (equation (1)) and Q representing the inhalative flow rate: (a) extrathoracic deposition, (b) tracheal deposition, (c) deposition in airway generation 1, (d) deposition in airway generation 2, (e) deposition in airway generation 3, (f) deposition in airway generation 4 and all following generations.

knowledge concerning the behavior of such fibrous particles in the human respiratory tract is of invaluable importance. Since about 20 years mathematical models have been created (e.g. [4, 11, 20]), which represent a reliable support for a complete decoding of fiber deposition in the lung airways and its effects on the lung tissue. In this contribution, deposition of variably shaped asbestos fibers was simulated by using two standard breathing conditions (sitting and lightwork breathing; [4]) as well as a wider spectrum of inhalative flow rates. The results obtained from the study may be regarded as very interesting in several aspects: First, it could be demonstrated that fibrous particles with a density of $2.8 \mathrm{~g}$ $\mathrm{cm}^{-3}$ are characterized by a lung deposition, which in parts differs significantly from that of comparable spheres. Second, deposition behavior of fibers is affected to a high extent by the cylindrical fiber diameter $\mathrm{d}_{\mathrm{p}}$, whereas fiber length has to be evaluated as a minor determinant for deposition. The second finding was already outlined in experimental studies, where rats were exposed to glass fibers with different length [9].

In general, deposition of asbestos fibers in the human respiratory tract is controlled by four deposition mechanisms, from which Brownian diffusion only affects sub-micron particles, whilst inertial impaction, interception, and sedimentation have a major influence on particles with $d_{p} \geq 1 \mu \mathrm{m}[11,30$, 31]. Furthermore, inertial impaction and interception are chiefly limited to the extrathoracic airways and the uppermost airway generations of the tracheobronchial tree, where airflow may be regarded as turbulent with Reynolds number being 
much greater than 1 [4]. Sedimentation, on the other side, mainly occurs in the intermediate and distal airway generations of the tracheobronchial tree that are characterized by a laminar airflow (low Reynolds number) due to a continuous flow splitting at the airway bifurcations. The balance of the four deposition mechanisms has important consequences for the total, regional, and generation-by-generation deposition of asbestos fibers: First, total deposition of $\mu \mathrm{m}$-sized fibers is clearly enhanced with respect to that of comparable spheres (Fig. 3) due to the increased effect of inertial impaction, interception, and sedimentation. Second, regional deposition is based on the same phenomenon, whereby fibers with $d_{p}=10$ $\mu \mathrm{m}$ are mainly accumulated in the mouth cavity, oropharynx, and larynx. Fibers with $d_{p}=0.1 \mu \mathrm{m}$ are chiefly deposited due to Brownian diffusion, giving them the opportunity to penetrate to the bronchiolar and alveolar lung compartment (note: Penetration depth depends upon the ratio between particle transport time through the airways and diffusion time.). Fibers with $\mathrm{d}_{\mathrm{p}}=1 \mu \mathrm{m}$ exhibit a somewhat indifferent deposition behavior, indicating similar effects of inertial impaction and interception on the one side and sedimentation on the other side [31]. Third, generation-by-generation deposition of fibers corresponds to the deposition mechanisms insofar, as the formation of a clear maximum indicates the predominance of a single deposition mechanism (e.g. Brownian diffusion in the case of fibers with $d_{p}=0.1 \mu \mathrm{m}$; Fig. 5a, d), whereas two or more deposition maxima indicate the involvement of more deposition mechanisms (e.g. fibers with $d_{p}=1 \mu \mathrm{m}$; Fig. 5b, e).

An interesting question concerns the modification of fiber deposition with increasing inhalative flow rate. As could be already shown for ideal spherical particles, any enhancement of both the breathing frequency and the tidal volume has two major effects $[4,30,31]$ : On the one side, inertial impaction and interception of large particles $(>1$ $\mu \mathrm{m})$ are significantly increased, causing a deposition concentration of these particles in the uppermost parts of the human respiratory tract. On the other side, Brownian diffusion mostly affecting sub-micron particles is subject to a noticeable dislocation to more distal regions of the pulmonary system. As demonstrated in Figs. $(4,5)$, these essential findings obtained for spheres may be also transferred to fibrous particles. The deviating behavior of fibers with $d_{p}=1 \mu \mathrm{m}$ again results from the circumstance that deposition of these particle class is controlled by more than one mechanism. Thereby, any increase of deposition by inertial impaction and interception in the upper airways leads to an obligatory decrease of deposition in the terminal airways, underlining the filter efficiency of the extrathoracic region and large bronchi.

This contribution confirms the widely accepted paradigm, according to which asbestos fibers and biopersistent fibers in general represent remarkable health hazards. Results of the comprehensive computations clearly showed that fibers with small cylindrical diameter $\left(\mathrm{d}_{\mathrm{p}}=0.1 \mu \mathrm{m}\right)$ have an enhanced ability to penetrate to the deepest parts of the lungs (i.e. respiratory bronchioli and alveoli), where they are subject to different clearance processes [7, 32, 33]. A certain fraction is stored in the lung parenchyma, where it may induce numerous lung diseases and, in the worst case, may act as severe carcinogen. Fibers with large cylindrical diameter $\left(d_{p}=10 \mu \mathrm{m}\right)$, on the other hand, are almost completely filtered in the uppermost parts of the human respiratory system, where most of them are cleared by fast clearance mechanisms such as the mucociliary escalator $[4,7]$. Nevertheless, also bronchial sites with increased accumulation of fibrous material (i.e. the carinal ridges of bronchial bifurcations) may be affected by pathological modifications, at the end of which stands the adenocarcinoma. An exception of this role is commonly represented by the trachea, which due to its vertical orientation (sitting and light work breathing) offers a minor target for particle deposition (Fig. 6). Further, continuous covering of the epithelial wall with a mucus layer and velocity of mucociliary clearance reach an optimum in the tracheal tube. Fibers with intermediate diameters $\left(d_{p}=1\right.$ $\mu \mathrm{m})$ may be regarded as most hazardous, as they are deposited in both the proximal and distal parts of the lungs and therefore may induce pathological changes in both regions.

\section{REFERENCES}

[1] International Agency for Research on Cancer (IARC). IARC monographs on the evaluation of carcinogenic risks to humans, Vol. 81. Man-made vitreous fibers. Lyon: IARC Press 2002; 81: pp. 1-418.

[2] Su WC, Cheng YS. Deposition of fiber in the human nasal airway. Aerosol Sci Technol 2005; 39: 888-901.

[3] Su WC, Cheng YS. Deposition of fiber in a human airway replica. J Aerosol Sci 2006; 37: 1429-41.

[4] International Commission on Radiological Protection (ICRP). Human respiratory tract model for radiological protection, Publication 66, Oxford: Pergamon Press: 1994; pp. 24(1-3).

[5] Muscat JE, Wynder EL. Cigarette smoking, asbestos exposure, and malignant mesothelioma. Cancer Res 1991; 51: 2263-67.

[6] Bianchi C, Bianchi T. Malignant mesothelioma: global incidence and relationship with asbestos. Ind Health 2007; 45: 379-87.

[7] Sturm R, Hofmann W. Mechanistic interpretation of the slow bronchial clearance phase. Radiat Prot Dosimetry 2003; 105: 10104.

[8] Travis WD, Travis LB, Devesa SS. Lung cancer. Cancer 1995; 75: 191-202.

[9] Lippmann M. Effects of fiber characteristics on lung deposition, retention, and disease. Environ Health Perspect 1990; 88: 311-17.

[10] Myojo T. Deposition of fibrous aerosol in model bifurcating tubes. J Aerosol Sci 1987; 18: 337-47.

[11] Sturm R, Hofmann W. A computer model for the simulation of fiber deposition in the human respiratory tract. Comput Biol Med 2006; 36: 1252-67.

[12] Timbrell V. Deposition and retention of fibers in human lung. Ann Occup Hyg 1982; 26: 347-69.

[13] Sussman RG, Cohen, BS, Lippmann M. Asbestos fiber deposition in human tracheobronchial cast. I. Experimental. Inhal Toxicol 1991; 3: 145-60.

[14] Berstein DM, Riego Sintes JM, Ersboell BK, Kunert J. Biopersistence of synthetic mineral fibers as a predictor of chronic intraperitoneal injection tumor response in rats. Inhal Toxicol 2001; 13: $851-75$.

[15] Stöber W. Dynamic shape factors of nonspherical aerosol particles. In: Mercer T, Ed. Assessment of airborne particles. Springfield: Charles C Thomas Publisher 1972; pp. 249-89.

[16] Kasper G. Dynamics and measurement of smokes. I Size characterization of nonspherical particles. Aerosol Sci Technol 1982; 1: 187-99.

[17] Dai YT, Yu CP. Alveolar deposition of fibers in rodents and humans. J Aerosol Med 1998; 11: 247-58.

[18] Virtanen A, Ristimäki J, Keskinen J. Method for measuring effective density and fractal dimension of aerosol agglomerates. Aerosol Sci Technol 2004; 38: 437-46

[19] Arsenijevic ZL, Grbavcic ZB, Garic-Grulovic RV, Zdanski FK. Determination of non-spherical particle terminal velocity using particulate expansion data. Powder Technol 1999; 103: 265-73.

[20] Koblinger L, Hofmann W. Monte Carlo modeling of aerosol deposition in human lungs. Part I: simulation of particle transport in a stochastic lung structure. J Aerosol Sci 1990; 21: 661-74.

[21] Slowik JG, Stainken K, Davidovits P, et al. Particle morphology and density characterization by combined mobility and aerody- 
namic diameter measurements. Part 2: application to combustiongenerated soot aerosols as a function of fuel equivalence Ratio. Aerosol Sci Technol 2004; 38: 1206-22.

[22] DeCarlo PF, Slowik JG, Worsnop DR, Davidovits P, Jimenez JL. Particle morphology and density characterization by combined mobility and aerodynamic diameter measurements. Part 1: theory. Aerosol Sci Technol 2004; 38: 1185-1205.

[23] Raabe OG, Yeh HC, Schum GM, Phalen RF. Tracheobronchial geometry: Human, dog, rat, hamster - A compilation of selected data from the project respiratory tract deposition models. Report LF-53. Albuquerque: Lovelace Foundation 1976.

[24] Haefeli-Bleuer B, Weibel ER. Morphology of the human pulmonary acinus. Anat Rec 1988; 220: 401-14.

[25] Koblinger L, Hofmann W. Analysis of human lung morphometric data for stochastic aerosol deposition calculations. Phys Med Biol 1985; 30: 541-56.

[26] Yeh HC, Schum GM. Models of human lung airways and their application to inhaled particle deposition. Bull Math Biol 1980; 42: 461-80.
[27] Asgharian B, Anjilvel S. Movement and deposition of fibers in an airway with steady viscous flow. Aerosol Sci Technol 1995; 22: 261-70.

[28] Cai FS, Yu CP. Inertial and interceptional deposition of spherical particles and fibers in abifurcating airway. J Aerosol Sci 1988; 19 679-88.

[29] Chen YK, Yu CP. Sedimentation of fibers from laminar flows in a horizontal circular duct. Aerosol Sci Technol 1991; 14: 343-7.

[30] Hofmann W, Koblinger L. Monte Carlo modeling of aerosol deposition in human lungs. Part II: deposition fractions and their sensitivity to parameter variations. J Aerosol Sci 1992; 21: 675-88.

[31] Hofmann W, Koblinger L. Monte Carlo modeling of aerosol deposition in human lungs. Part III: comparison with experimental data J Aerosol Sci 1992; 23: 51-63.

[32] Heesterberg TW, Hart GA. Synthetic vitreous fibers: a review of toxicology research and its impact on hazard classification. Crit Rev Toxicol 2001; 31: 1-53.

[33] Kamstrup O, Ellehauge A, Collier CG, Davies JMG. Carcinogenicity studies after intraperitoneal injection of two types of stone wool fibres in rats. Ann Occup Hyg 2002; 46: 135-42.

(C) Robert Sturm; Licensee Bentham Open.

This is an open access article licensed under the terms of the Creative Commons Attribution Non-Commercial License (http://creativecommons.org/licenses/bync/3.0/) which permits unrestricted, non-commercial use, distribution and reproduction in any medium, provided the work is properly cited. 